\title{
PERIOD CHANGES OF RR LYRAE STARS IN \\ GLOBULAR CLUSTERS
}

\author{
B. SZEIDL
}

Konkoly Observatory, Budapest

\begin{abstract}
The dependence of the period changes of RR Lyrae stars, especially in globular clusters, on the evolutionary track through the instability strip has still found no explanation. The present paper is an attempt to give a review of the observed phenomena.
\end{abstract}

Since Martin's (1938) discovery that the periods of the RRab stars in Omega Centauri are predominantly increasing, many studies have been made on period changes of a great number of RR Lyr stars in different clusters, mostly at the Sternberg Institute and the Asiago, Toronto and Budapest Observatories. In the early studies it was thought that the $\mathrm{O}-\mathrm{C}$ diagrams were simply accumulated evolutionary changes in the periods. Little wonder then that the deviations of the epochs of variable stars from the linear elements were represented by second order equations, where the coefficients of the second order terms (called Beta) were supposed to describe the progressive changes in the periods. It was hoped that these coefficients would give some informations on the rate and direction, of evolution of horizontal branch stars through the instability strip and provide a strong test of the theory of stellar evolution.

Theoreticians have generally found that the rate at which a model passes through the instability strip during core helium burning (Iben and Rood, 1970) is some orders of magnitude slower than the rate of period changes observed for many of the cluster variables. This apparent discrepancy and the very complex structure of some of the O-C diagrams suggest that some kind of random 'noise' (of considerable amount sometimes) is superimposed on those secular variations of the period which we expect to exist as a result of evolutionary changes in the mean stellar radius.

Almost ten years ago at the 3rd Bamberg Colloquium, Balázs-Detre and Detre (1966) investigated the probability structure of the O-C diagram making the simple assumptions that the successive period jumps are independent random variables with zero mean and that the stochastic process is stationary. In this case the structure of the O-C diagram depends only on the variance of the period fluctuation and the O-C diagram consists of cycles of different lengths and amplitudes.

Apart from the nature of the slow secular evolutionary changes, a knowledge of the character of period variation is also of basic importance to theoretical investigations. There is presently doubt about the character of the changes: whether the periods are relatively constant for long time intervals followed by brief intervals of spontaneous changes (Goranskij et al., 1973) or whether the variations are relatively smooth.

Although the observations of the RR Lyrae stars in some globular clusters embrace a time interval of more than half a century already, it is almost impossible to 


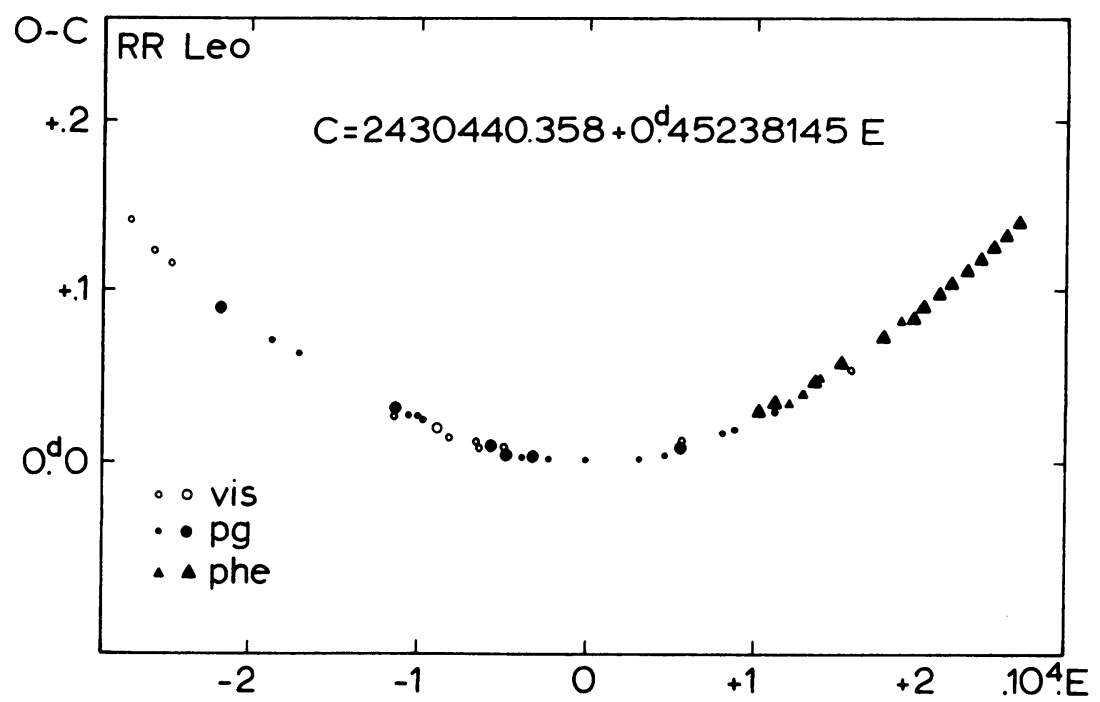

Fig. 1. O-C diagram for RR Leo.

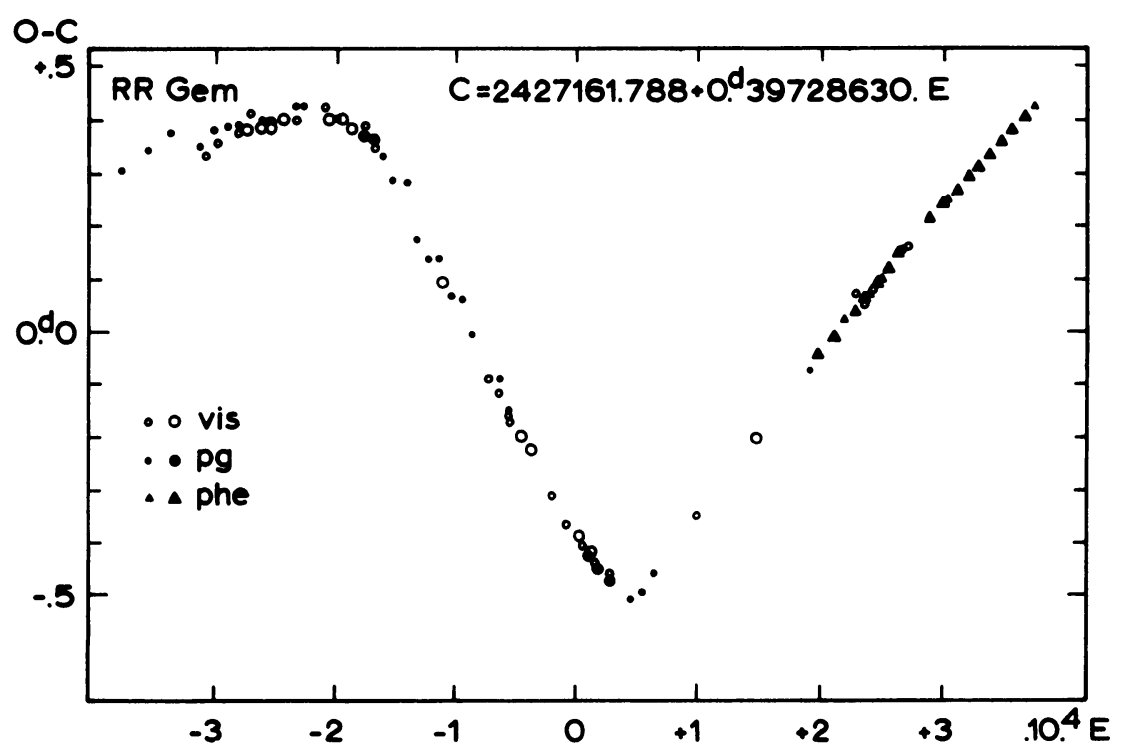

Fig. 2. O-C diagram for RR Gem.

settle the question regarding the character of the period changes. Unfortunately, the observations are very unevenly distributed during the time interval and the uncertainty is fairly large. I think that the study of the O-C diagrams of field RR Lyrae stars can help. Figures 1 and 2 show the O-C diagram of two well observed stars, RR Leo and RR Gem. These two stars which are still on our programme, have been extensively observed photoelectrically during the last 20 years. 
The period of RR Leo has increased smoothly, but the rate of change is not at all constant. On the other hand, the period of RR Gem changed suddenly around J.D. 2428900 within a brief interval. Around this epoch RR Gem showed the Blažkoeffect. After J.D. 2428900 the O-C diagram can be well approximated by two discontinuous lines, although a smooth change cannot be completely excluded. I assume that there is no essential difference between field RR Lyrae stars of low metal abundance and cluster variables so we can come to the conclusion that generally both smooth and sudden changes in the periods occur. Nevertheless, in many cases an approximation by linear elements can be suitable and the period jumps and time intervals between the breaks may prove to be good quantities for statistical analysis as measures of instability of the periods (cf. Goranskij et al., 1973).

All things considered the period changes seem to be a very important characteristic feature of RR Lyrae stars and their correct interpretation is one of the main outstanding problems of the study of cluster variables.

In the past several years at the Konkoly Observatory we have completed studies of the period changes of cluster variables in M 3 (Szeidl, 1965, 1973) and in M 15 (Barlai, 1975). As these two clusters belong to different Oosterhoff-types (M 3 to type I, M 15 to type II) their comparison seemed very promising. We now have the $\mathrm{O}-\mathrm{C}$ diagrams of 116 RR Lyrae stars in M 3 and 44 RR Lyrae stars in M 15, covering a time interval of about 70 years.

It is very striking that if we follow tradition and approximate the O-C's by quadratic forms we meet with less success in the case of the cluster variables in $M 15$ than in M 3. Among $101 \mathrm{RRab}$ stars in M 3 the O-C diagram of $42(\sim 40 \%)$ could be approximated more or less by quadratic form, while for M 15 this is the case for only five of the 20 investigated RRab stars $(\sim 25 \%)$. Three of the 15 RRc stars in M 3 $(\sim 20 \%)$ and none of the $24 \mathrm{RRc}$ stars $(\sim 0 \%)$ studied in M 15 have $\mathrm{O}-\mathrm{C}$ diagrams which can be fitted more or less acceptably by parabolas. This kind of investigation has also convinced us that the Beta quantities are very deceptive ones.

In order to say something about the period changes of the variables in M 3 and M 15 we defined the 'average periods' $P_{1}$ and $P_{2}$ for the time intervals J.D. 2415000 2425000 and J. D. $2430000-2437000$, respectively in such a way that these parts of the O-C diagrams were approximated by straight lines. From these periods we derived the rate of period change $\triangle P / P \Delta E$ in units of day/day. If we accept that the period changes are apparent and caused by random fluctuations this quantity has not too much meaning for a single star; it may be, however, useful for statistical investigations and for comparison between clusters. Such quantities could be defined for 40 RR Lyrae stars in M 15 and 112 RR Lyrae stars in M 3. We have not excluded (as many people have) the RR Lyrae stars with Blažko-effect from our investigations. Certainly, the changes in the period of stars with Blažko-effect are in most cases very large but by omitting them one may perhaps introduce selection effects.

Figure 3 shows the distribution of period changes observed for both RRab and RRc stars in M 3 and M 15. In Figure 4 the period variation is plotted against period. It is striking that the spread of the period changes of the RRc type stars is smaller 


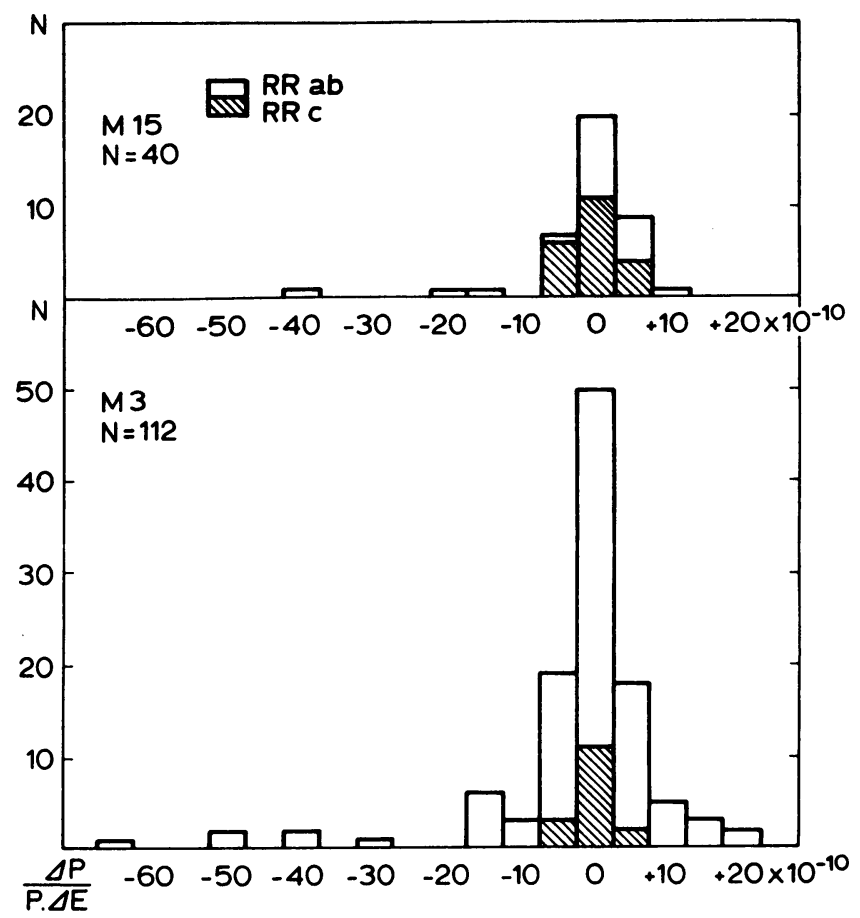

Fig. 3. Distribution in number versus rate of period change for variables in $\mathbf{M} 3$ and $\mathbf{M} 15$.

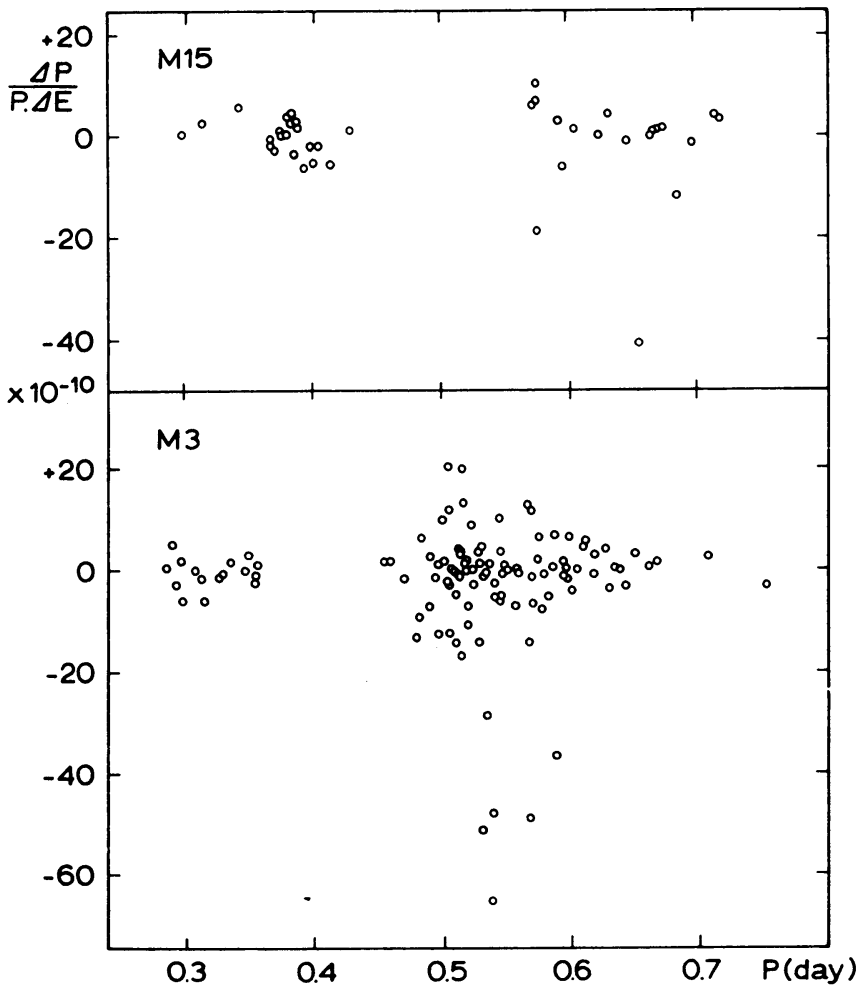

Fig. 4. Rate of period change versus period for variables in $M 3$ and $M 15$. 
than that of the RRab stars. Till now the opposite has been stated by the observers. The truth is, however, that the c-type stars were usually neglected in the investigations. We also see that there are some large negative values in the rate of period variations but we would be mistaken if we interpreted this as a general trend of period decrease. The O-C diagrams shown in Figures 5 and 6 are examples of stars for which we could not find a rate of period change in the way defined above; these show large, sudden, positive changes (more than $10 \mathrm{~s}$ ) in their periods.

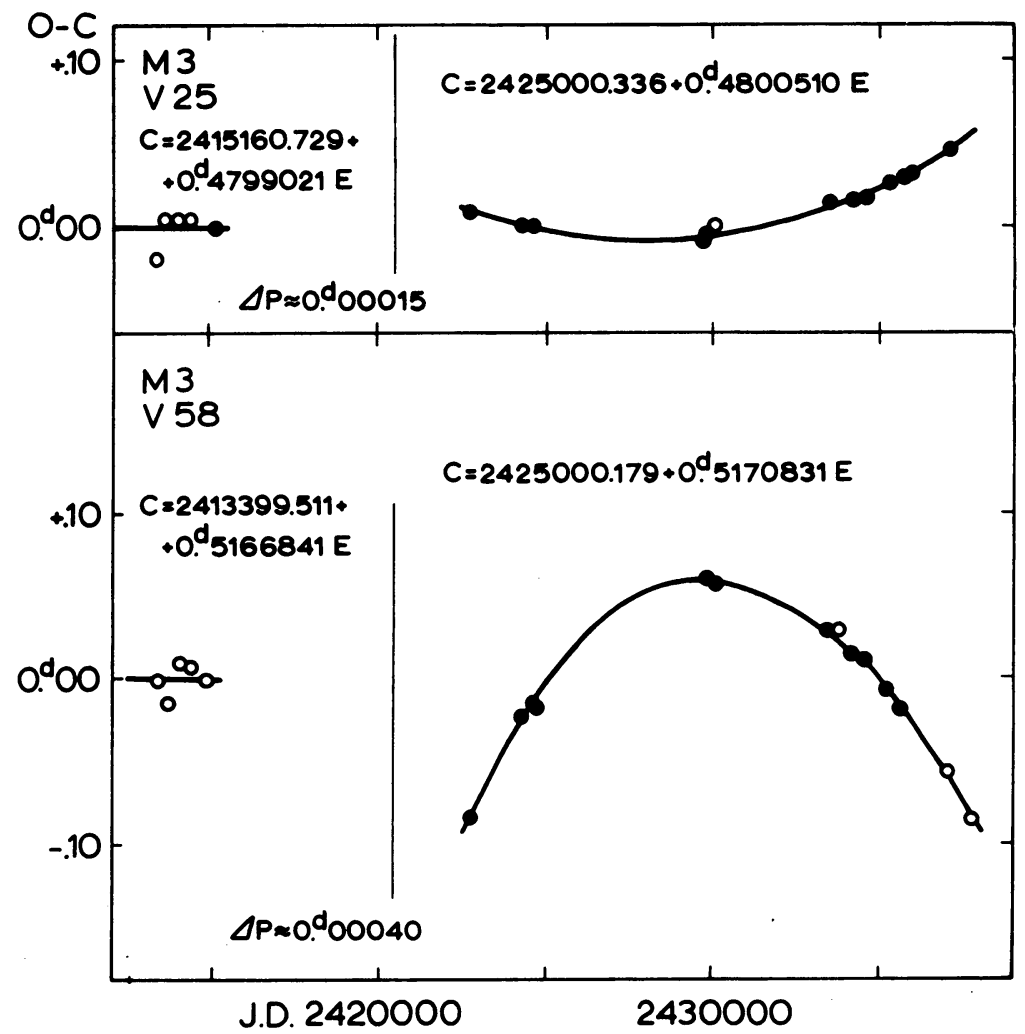

Fig. 5. O-C diagrams for the variables No. 25 and No. 58 in M 3.

It was originally hoped that the period variations would be found to depend on position in the colour-magnitude diagram. These expectations are not quite fulfilled. Figure 7 shows the colour-magnitude diagram for the RR Lyrae stars in M 3 using Sandage's (1959) colours and magnitudes. The arrows indicate the direction and rate of the period changes. From this figure we could not find any connection between the position of a star and its period change.

A similar diagram has been constructed for the RR Lyrae stars of M 15. On the period-median brightness diagram (Figure 8) we also indicate the direction and rate of period changes. Only the position of Var. 49 is worth mentioning. This star is 
B. SZEIDL

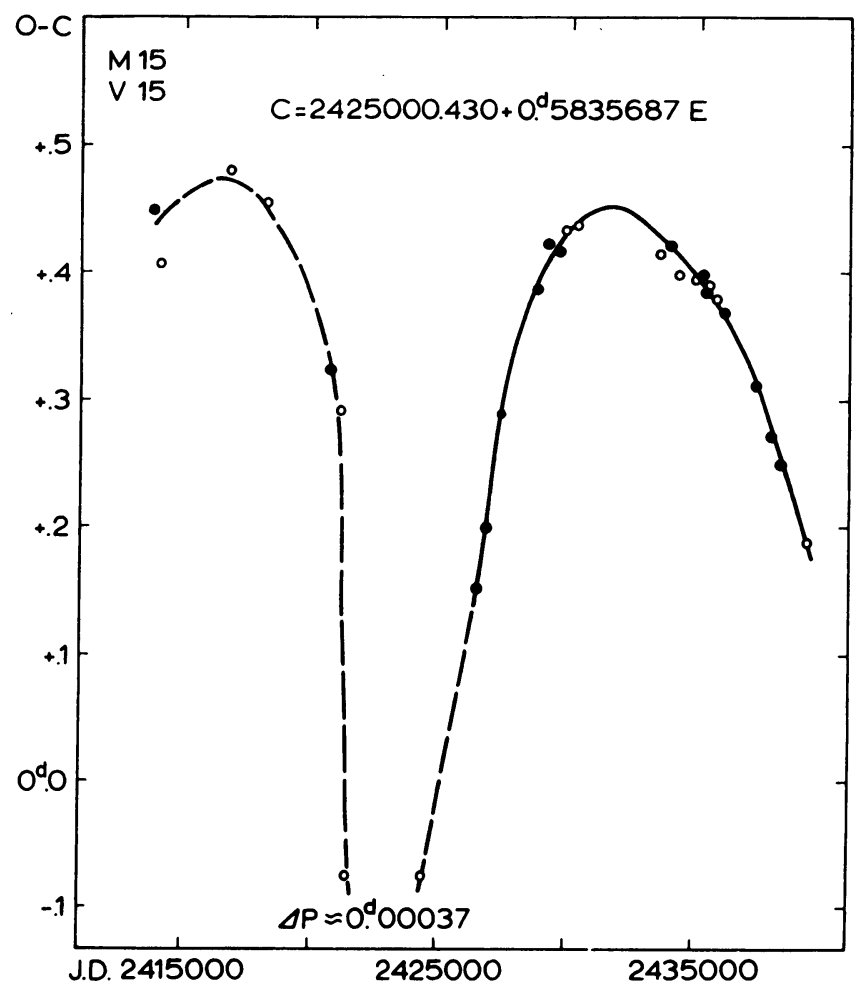

Fig. 6. O-C diagram for the variable No. 15 in $M 15$.

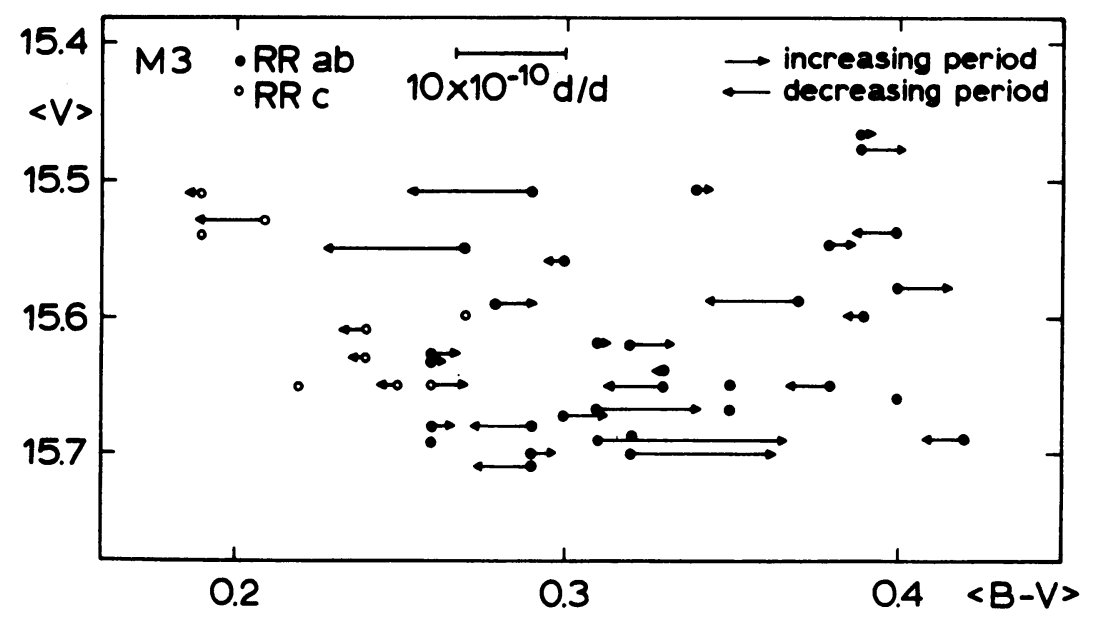

Fig. 7. Colour-magnitude diagram for RR Lyrae variables in M 3 (Sandage, 1959). The arrows indicate the direction and rate of period changes. 
brighter than the others. The star is either a foreground RR Lyrae variable or it is related to those short period variables that are brighter than the ordinary RR Lyrae stars by 0.5 to $0.75 \mathrm{mag}$. and are typical of the Sculptor type systems. This kind of variable has not yet been observed among the variables in the galactic globular clusters (van Agt, 1972).

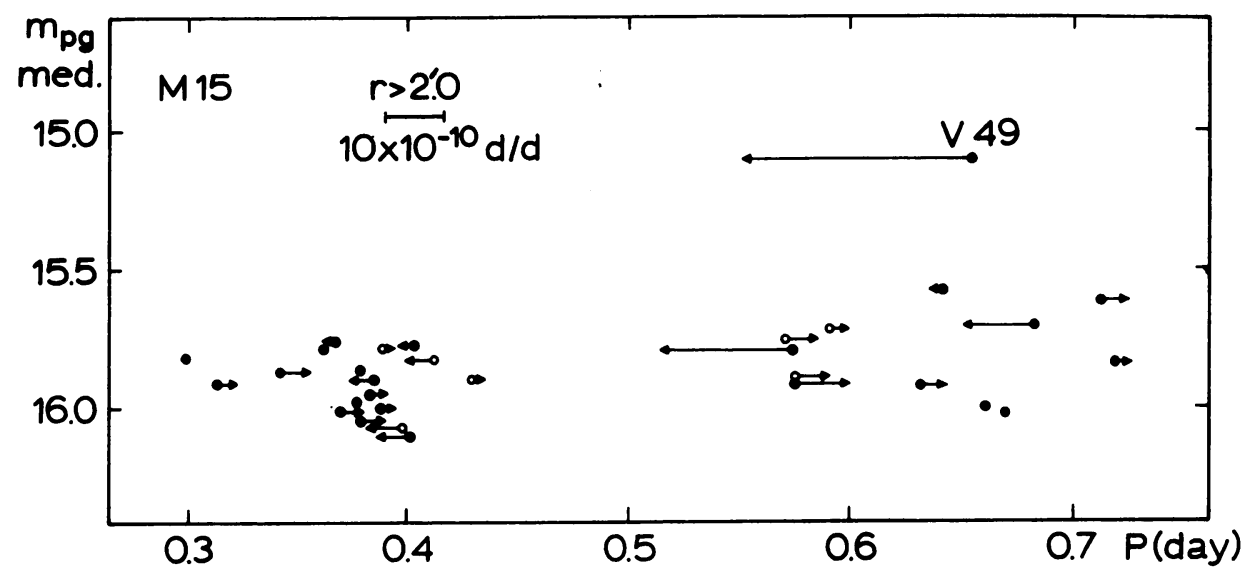

Fig. 8. Period-median brightness diagram for variables in $M$ 15. The arrows indicate the direction and rate of period changes.

A useful quantity that can be derived from observations may be $N_{+} / N_{-}=$(number with increasing period)/(number with decreasing period). This quantity is for both M 3 and M 15 near one, which is not in full agreement with evolutionary theories.

Comparing our results obtained for M 3 and M 15 with those obtained for other clusters (M 5: Coutts and Sawyer Hogg; M 14: Wehlau and Potts) we come to the final conclusion that at present it is impossible to attach any evolutionary significance to the observed period changes.

Omega Centauri is the only exception in which there may be present some evidence for evolution effects (Belserene, 1973). This cluster, however, shows a remarkable anomaly at the long end of the RR Lyrae periods: it contains more than twice as many RR Lyrae stars with periods longer than 0.75 days as all other globular clusters combined. Perhaps this fact is connected with the detected evolutionary changes.

Summing up what has been said the random variations of the period are a common and normal characteristic of the cluster variables. For their correct interpretation long series of continuous observations of the RR Lyrae stars in globular clusters are necessary.

\section{Acknowledgements}

I am very much indebted to Dr K. Barlai who has generously put at my disposal her results on M 15. I thank Prof. L. Detre and Dr W. Osborn for helpful remarks and suggestions. 


\section{References}

van Agt, S.: 1972, Dudley Observatory Reports, No. 4, p. 101.

Balázs-Detre, Julia and Detre, L.: 1966, Kleine Veröff. Bamberg 4, Nr. 60, p. 184.

Barlai, K.: 1975, Mitt. Sternw. Ungar. Akad. Wiss. Budapest-Szabadsághegy (to be published).

Belserene, E. P.: 1973, in J. D. Fernie (ed.), Variable Stars in Globular Clusters and in Related Systems,

D. Reidel Publ. Co., Dordrecht, p. 105.

Coutts, C. M. and Sawyer Hogg, H.: 1969, Publ. David Dunlap Obs. 3, No. 1, p. 1.

Goranskij, V. P., Kukarkin, B. V., and Samus, N. N.: 1973, in J. D. Fernie (ed.), Variable Stars in Globular Clusters and in Related Systems, p. 101.

Iben, I. jr. and Rood, R. T.: 1970, Astrophys. J. 161, 587.

Martin, W. Chr.: 1938, Ann. Sterrew. Leiden 17, Part 2, p 1.

Sandage, A. R.: 1959, Astrophys. J. 129, 596.

Szeidl, B.: 1965, Mitt. Sternw. Ungar. Akad. Wiss., Budapest-Szabadsághegy, Nr. 58.

Szeidl, B.: 1973, Mitt. Sternw. Ungar. Akad. Wiss., Budapest-Szabadsághegy, Nr. 63.

Wehlau, A. and Potts, N.: 1973, in J. D. Fernie (ed.), Variable Stars in Globular Clusters and in Related Systems, p. 95.

\section{DISCUSSION}

C. M. Coutts: From your investigation it appears that among the stars of RRa type in M 15, there is a tendency for the periods to increase (similar to $\omega$ Centauri, another metal-poor cluster). In $M 3$, on the other hand, there are a large number of RRa type stars with decreasing period. This is similar to the case of M 5, which is a cluster with metal abundance similar to M 3. Do you think this is significant?

B. Szeidl: Certainly there are fewer RRab stars with decreasing period in M 15 than those with increasing period. But the number of ab stars investigated in $M 15$ is too few; no, I think that we cannot regard this tendency as significant.

J. Kreiner: What do you think about the influence of gravitational acceleration (theory of Dr Kordylewski) on the O-C diagram in globular clusters.

$B$. Szeidl: This influence is very small.

B. Rutily: Did you also notice variations in the periods of RR Lyrae variables of the galactic halo?

B. Szeidl: Yes. We have about twenty RR Lyrae variables of the galactic halo on our programme, and we observed changes in their periods as well as those in globular clusters.

$W$. Dziembowski: In the case of M 3 there is a maximum of absolute values of rates of period changes at a certain narrow range of periods, visible on your slide. Is this maximum statistically significant?

B. Szeidl: This is a consequence of the fact that most stars are located in this range of periods. 